\title{
Fill Rate Estimation in Periodic Review Policies with Lost Sales Using Simple Methods
}

\author{
Manuel Cardós (iD), Ester Guijarro Tarradellas (iD), Eugenia Babiloni Griñón(iD \\ Universidad Politécnica de Valencia (Spain) \\ mcardos@,doe.upv.es, esguitar@,upvnet.upv.es,mabagri@,doe.upv.es
}

Received: July 2016

Accepted: October 2016

\section{Abstract:}

Purpose: The exact estimation of the fill rate in the lost sales case is complex and time consuming. However, simple and suitable methods are needed for its estimation so that inventory managers could use them.

Design/methodology/approach: Instead of trying to compute the fill rate in one step, this paper focuses first on estimating the probabilities of different on-hand stock levels so that the fill rate is computed later.

Findings: As a result, the performance of a novel proposed method overcomes the other methods and is relatively simple to compute.

Originality/value: Existing methods for estimating stock levels are examined, new procedures are proposed and their performance is assessed.

Keywords: fill rate, lost sales, periodic review policy 


\section{Introduction and Literature Review}

Inventory models in the lost sales context are harder to formulate than backordering models because the loss of unfulfilled demand is more difficult to represent and manage as pointed out by Bijvank, Huh, Janakiraman and Kang (2014) and Zipkin, 2008a, 2008b. However, the lost sales case is quite common in sectors and industries where customers are not prepared for waiting when a stockout occurs as for example in the retail industry or in ecommerce.

Three papers can be found in the lost sales context that provide a way to estimate the probabilities of the on-hand stock levels although their aim is quite different. For example, Cardós, Miralles and Ros (2005) propose the only known method that provides an exact method to compute the Cycle Service Level in a discrete lost sales demand context and also provides the exact on-hand stock levels. This method, named Exact Method further on, is based on the probability transition matrixes of the on-hand stock levels from the beginning of each cycle to its end; the convergence of the resulting Markov chain provides the on-hand probability vector at the beginning of the cycle. Cardós and Babiloni (2011) derive an approximation of the Cycle Service Level assuming that there are no stockouts during the lead time so that the probability vector of the on-hand stock levels at the beginning of the cycle is computed as in the backordering case. This method, named M1 method henceforth, can be used for any discrete demand distribution. Bijvank and Johansen (2012) propose an approximation procedure for computing the average on-hand stock when demand is compound Poisson. This method, named B\&J method below, basically starts from the on-hand stock probabilities as in the backlog case but multiplied by a correction factor in order to provide the average stock obtained applying the Little's Law.

The aim of this paper is to derive and evaluate procedures to compute the fill rate based on: (a) an estimation of the on-hand stock levels at the beginning of the cycle; (b) in the lost sales context and with any discrete demand distribution; and (c) easy to implement in practical environments.

The rest of the paper is organized as follows. Section 2 presents the basic notation and assumptions, Section 3 proposes four new methods to estimate the on-hand stock level probabilities at the beginning of the cycle and Section 4 evaluates the performance of the existing and new methods when used to estimate the fill rate. Finally, Section 5 highlights the conclusions of this work. 


\section{Notation and Assumptions}

Periodic review policies place replenishment orders every $\mathrm{R}$ time periods such that the on-hand stock plus outstanding orders reach the order-up-to level S. The order is received L time periods later. Figure 1 shows an example (a) where no stockout occurs and another example (b) showing a stockout. The notation in Figure 1 and in the rest of the paper is as follows:

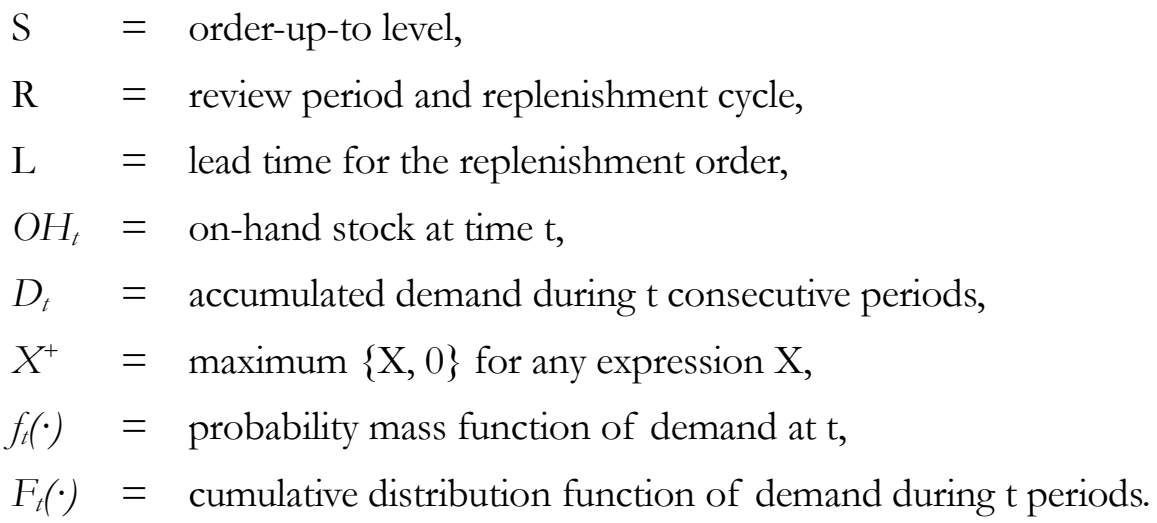

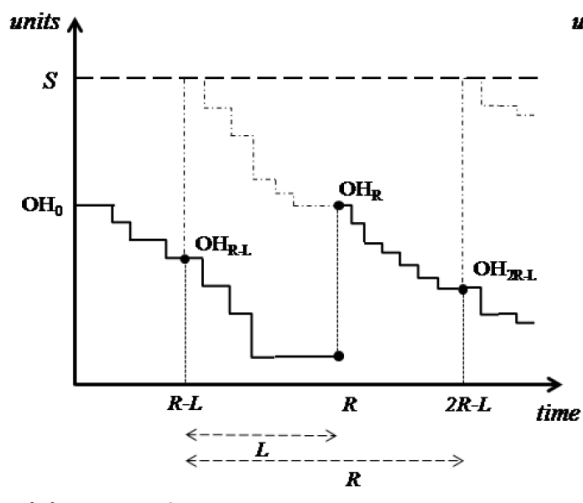

(a) Non stockout case

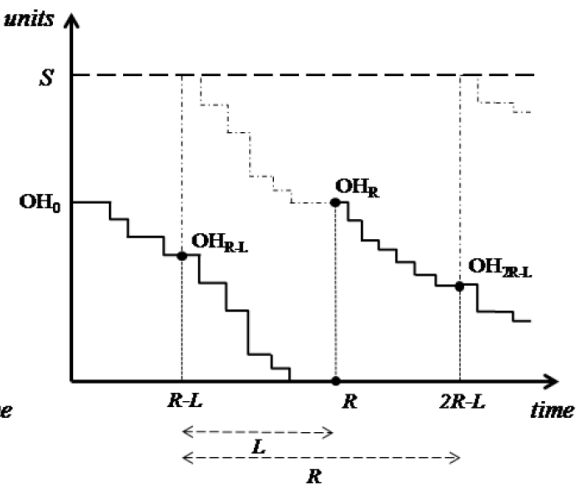

(b) Stockout case

Figure 1. Periodic review system with lost sales with and without stockout

General assumptions of this paper are: (i) time is discrete and is organized in a numerable and infinite succession of equally spaced instants; (ii) the lead time and the review period are constant and known; (iii) there is never more than one outstanding order, leading to $\mathrm{L}<\mathrm{R}$; (iv) the replenishment orders are received at the end of the period; (v) demand is fulfilled with the on-hand stock at the beginning of the period; (vi) demand is stationary and i.i.d. and follows any discrete distribution function; and (vii) unfulfilled demand is lost. 


\section{Proposed Methods}

\subsection{Fill Rate Estimation}

Guijarro, Cardós and Babiloni (2012) compute the fill rate using a classical approach such as

$$
\beta=1-\frac{\sum_{i=0}^{S} P\left(O H_{R}=i\right) \cdot \sum_{j=i+1}^{\infty}(j-i) \cdot f_{R}(j)}{\sum_{j=1}^{\infty} j \cdot f_{R}(j)}
$$

However, we use the expression below because of its superior numerical stability and adherence to the fill rate definition itself

$$
\beta=\frac{\sum_{i=0}^{S} P\left(O H_{R}=i\right) \cdot \sum_{j=1}^{i}\left\{j \cdot f_{R}(j)+i\left[1-F_{R}(i)\right]\right\}}{\sum_{j=1}^{\infty} j \cdot f_{R}(j)}
$$

\subsection{On-hand Estimation Methods}

M1 method assumes no stockout during the cycle, so the on-hand stock probabilities at the beginning of the cycle are

$$
\begin{aligned}
& O H_{R-L}=\left[O H_{0}-D_{R-L}\right]^{+} \\
& O H_{R}=\left[O H_{0}-D_{R}\right]^{+}+S-O H_{R-L}=S-D_{L} \\
& P\left(O H_{R}=i\right)=\left\{\begin{array}{cc}
f_{L}(S-i) & i=1, \ldots, S \\
1-F_{L}(S-1) & i=0
\end{array}\right.
\end{aligned}
$$

These probabilities can be expressed as a vector

$$
\overline{P\left(O H_{1}\right)}=\left(1-F_{L}(S-1) \quad f_{L}(S-1) \quad \ldots \quad f_{L}(0)\right)
$$

M2 method, the first proposed method, is the opposite of M1 method because now we assume that there is a stockout as soon as possible, before the stock is reviewed

$$
\begin{gathered}
O H_{R-L}=\left[O H_{0}-D_{R-L}\right]^{+}=0 \Rightarrow O H_{R}=S \\
\overline{P\left(\mathrm{OH}_{2}\right)}=\left(\begin{array}{llll}
0 & \ldots & 0 & 1
\end{array}\right)
\end{gathered}
$$


Based on M1 and M2 methods, M3 method proposes another estimate of the probability vector based on the probability of occurring the assumptions of $M 2$

$$
\begin{gathered}
P\left(\mathrm{OH}_{0}-D_{R-L} \leq 0\right)=P\left(D_{R-L} \geq S\right)=1-F_{R-L}(S-1)=\beta \\
\overline{P\left(\mathrm{OH}_{3}\right)}=\beta \cdot \overline{P\left(\mathrm{OH}_{2}\right)}+(1-\beta) \cdot \overline{P\left(\mathrm{OH}_{1}\right)}
\end{gathered}
$$

M4 method is based on improving this idea using the probability of $M 1$ assumptions

$$
\begin{aligned}
P\left(\mathrm{OH}_{0} \geq D_{R}\right) & =\sum_{i=0}^{S} P\left(O H_{0} \geq D_{R}\right) P\left(O H_{0}=i\right)= \\
& =\sum_{i=1}^{S} F_{R}(i) \cdot f_{L}(S-i)+F_{R}(0) \cdot\left[1-F_{L}(S-1)\right]=\alpha \\
\overline{P\left(\mathrm{OH}_{4}\right)} & =\frac{\beta}{\alpha+\beta} \cdot \overline{P\left(O H_{2}\right)}+\frac{\alpha}{\alpha+\beta} \cdot \overline{P\left(O H_{1}\right)}
\end{aligned}
$$

Finally, M5 method simplifies the calculation of $a$ and $\beta$ assuming that $a=\beta$ and $a+\beta=1$ so that

$$
\overline{P\left(\mathrm{OH}_{5}\right)}=\frac{1}{2} \cdot \overline{P\left(\mathrm{OH}_{2}\right)}+\frac{1}{2} \cdot \overline{P\left(\mathrm{OH}_{1}\right)}
$$

\section{Experimental Evaluation}

The rationale for every method is quite different, so it is necessary to assess their accuracy in terms of their deviations from the exact fill rate. We perform an extensive experiment including smooth, intermittent, lumpy and erratic demand but also a wide combination of stock policy parameters as seen in Table 1. This dataset provides 12,348 cases whose fill rate estimates are represented in Figure 2.

\begin{tabular}{|l|l|l|}
\hline Demand distribution \\
\hline Poisson & $\lambda$ & $0.01,0.1,0.5,1,2,5,10$ \\
\hline \multirow{2}{*}{ Negative Binomial } & $r$ & $0.1,0.25,0.5,0.75,1,1.25,1.5,3$ \\
\cline { 2 - 3 } & $\theta$ & $0.1,0.25,0.3,0.4,0.5,0.75,0.9$ \\
\hline Inventory system & $2,3,5,10,15,20,30$ \\
\hline R & $1,3,5,10,15,20$ \\
\hline L & $1,3,5,10,15,20,30$ \\
\hline $\mathrm{S}$ &
\end{tabular}

Table 1. Dataset of the experiment 

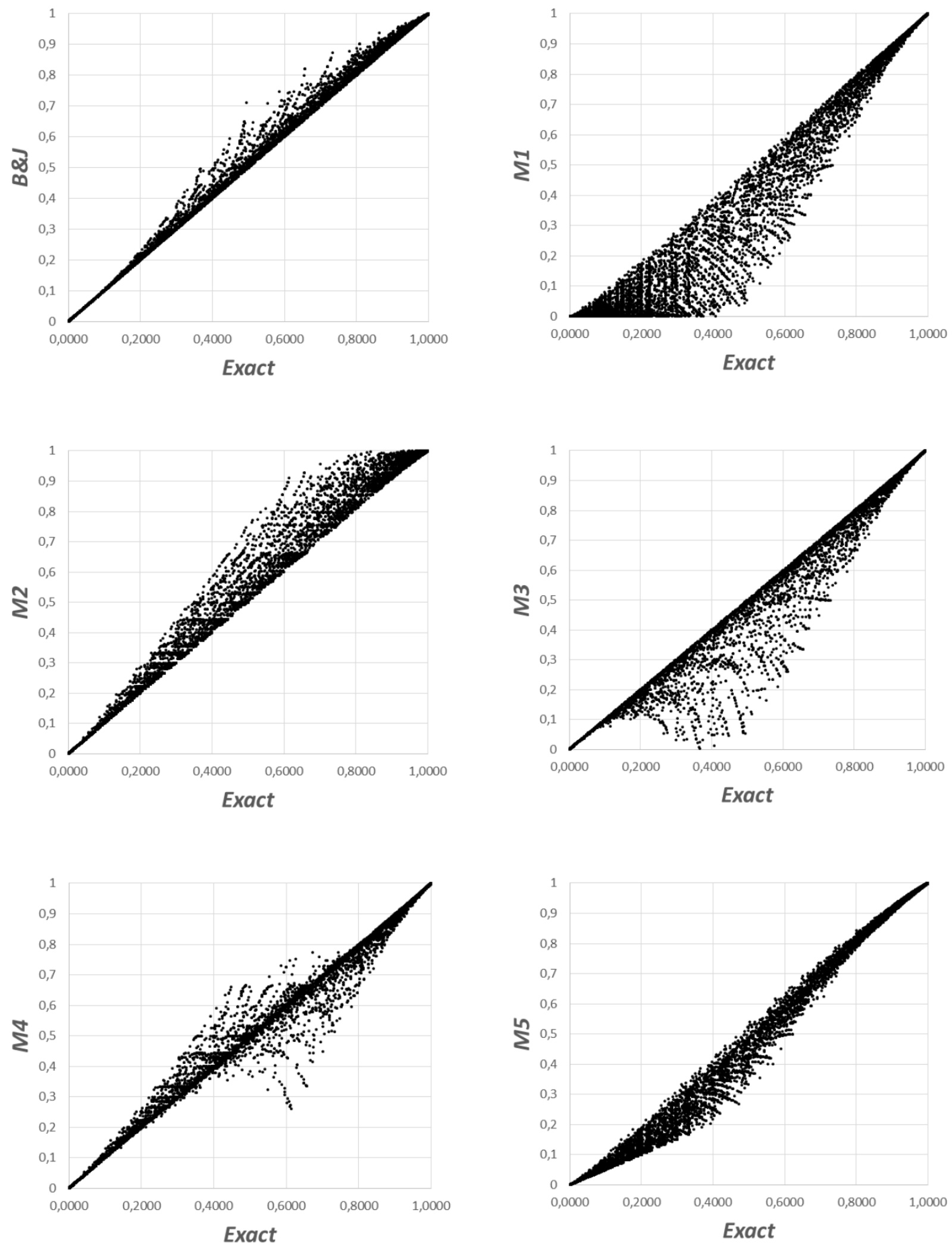

Figure 2. Fill rate estimates against the exact value for the considered methods

Additionally, Table 2 analyze the average, standard, maximum and minimum deviations for the estimation methods. This analysis just includes cases with a fill rate between 0.50 and 0.99 because cases out of this range have no practical interest. 


\begin{tabular}{|l|l|l|l|l|r|r|}
\hline FR & B\&J & M1 & M2 & M3 & \multicolumn{1}{c|}{ M4 } & \multicolumn{1}{c|}{ M5 } \\
\hline $0.50-0.55$ & 0.0238 & -0.1689 & 0.0707 & -0.0813 & 0.0003 & -0.0491 \\
\hline $0.55-0.60$ & 0.0271 & -0.1557 & 0.0803 & -0.0867 & -0.0185 & -0.0377 \\
\hline $0.60-0.65$ & 0.0250 & -0.1303 & 0.0721 & -0.0696 & -0.0288 & -0.0291 \\
\hline $0.65-0.70$ & 0.0271 & -0.1124 & 0.0859 & -0.0722 & -0.0422 & -0.0132 \\
\hline $0.70-0.75$ & 0.0278 & -0.0936 & 0.0824 & -0.0632 & -0.0426 & -0.0056 \\
\hline $0.75-0.80$ & 0.0232 & -0.0647 & 0.0717 & -0.0450 & -0.0363 & 0.0035 \\
\hline $0.80-0.85$ & 0.0208 & -0.0474 & 0.0684 & -0.0368 & -0.0316 & 0.0105 \\
\hline $0.85-0.90$ & 0.0153 & -0.0267 & 0.0505 & -0.0208 & -0.0193 & 0.0119 \\
\hline $0.90-0.95$ & 0.0100 & -0.0122 & 0.0349 & -0.0105 & -0.0103 & 0.0113 \\
\hline $0.95-0.99$ & 0.0016 & -0.0011 & 0.0063 & -0.0010 & -0.0010 & 0.0026 \\
\hline
\end{tabular}

(a) Average Deviation

\begin{tabular}{|l|l|l|l|l|r|r|}
\hline FR & B\&J & M1 & M2 & M3 & \multicolumn{1}{c|}{ M4 } & M5 \\
\hline $0.50-0.55$ & 0.0279 & 0.1042 & 0.0639 & 0.0969 & 0.0501 & 0.0417 \\
\hline $0.55-0.60$ & 0.0316 & 0.1004 & 0.0708 & 0.1022 & 0.0644 & 0.0379 \\
\hline $0.60-0.65$ & 0.0289 & 0.0883 & 0.0709 & 0.0890 & 0.0771 & 0.0328 \\
\hline $0.65-0.70$ & 0.0309 & 0.0753 & 0.0658 & 0.0783 & 0.0669 & 0.0258 \\
\hline $0.70-0.75$ & 0.0280 & 0.0621 & 0.0592 & 0.0651 & 0.0581 & 0.0211 \\
\hline $0.75-0.80$ & 0.0200 & 0.0437 & 0.0523 & 0.0463 & 0.0446 & 0.0141 \\
\hline $0.80-0.85$ & 0.0192 & 0.0333 & 0.0423 & 0.0347 & 0.0319 & 0.0120 \\
\hline $0.85-0.90$ & 0.0127 & 0.0181 & 0.0309 & 0.0189 & 0.0187 & 0.0090 \\
\hline $0.90-0.95$ & 0.0086 & 0.0089 & 0.0196 & 0.0093 & 0.0092 & 0.0060 \\
\hline $0.95-0.99$ & 0.0028 & 0.0021 & 0.0085 & 0.0020 & 0.0020 & 0.0032 \\
\hline
\end{tabular}

(b) Standard Deviation

\begin{tabular}{|l|l|l|r|r|r|r|}
\hline FR & B \&J & M1 & M2 & M3 & \multicolumn{1}{c|}{ M4 } & \multicolumn{1}{c|}{ M5 } \\
\hline $0.50-0.55$ & 0.1334 & -0.0076 & 0.2618 & 0.0000 & 0.1437 & 0.0378 \\
\hline $0.55-0.60$ & 0.1607 & -0.0074 & 0.2605 & 0.0000 & 0.1434 & 0.0437 \\
\hline $0.60-0.65$ & 0.1311 & -0.0053 & 0.2966 & 0.0000 & 0.1361 & 0.0396 \\
\hline $0.65-0.70$ & 0.1641 & -0.0066 & 0.2700 & 0.0000 & 0.0996 & 0.0415 \\
\hline $0.70-0.75$ & 0.1387 & -0.0049 & 0.2555 & 0.0000 & 0.0379 & 0.0434 \\
\hline $0.75-0.80$ & 0.0982 & -0.0025 & 0.2019 & 0.0000 & 0.0050 & 0.0416 \\
\hline $0.80-0.85$ & 0.0933 & -0.0007 & 0.1756 & 0.0000 & 0.0000 & 0.0429 \\
\hline $0.85-0.90$ & 0.0727 & -0.0006 & 0.1256 & 0.0000 & 0.0000 & 0.0329 \\
\hline $0.90-0.95$ & 0.0411 & -0.0001 & 0.0883 & 0.0000 & 0.0000 & 0.0252 \\
\hline $0.95-0.99$ & 0.0220 & 0.0000 & 0.0434 & 0.0000 & 0.0000 & 0.0157 \\
\hline
\end{tabular}

(c) Maximum Deviation

\begin{tabular}{|l|c|c|c|c|r|r|}
\hline FR & B\&J & M1 & M2 & M3 & M4 & M5 \\
\hline $0.50-0.55$ & 0.0000 & -0.4221 & 0.0000 & -0.3946 & -0.2257 & -0.1766 \\
\hline $0.55-0.60$ & 0.0000 & -0.3903 & 0.0003 & -0.3885 & -0.2885 & -0.1664 \\
\hline $0.60-0.65$ & 0.0000 & -0.3598 & 0.0031 & -0.3542 & -0.3541 & -0.1223 \\
\hline $0.65-0.70$ & 0.0000 & -0.3231 & 0.0009 & -0.3231 & -0.3230 & -0.0851 \\
\hline $0.70-0.75$ & 0.0000 & -0.2524 & 0.0028 & -0.2524 & -0.2523 & -0.0848 \\
\hline $0.75-0.80$ & 0.0000 & -0.1849 & 0.0050 & -0.1849 & -0.1849 & -0.0266 \\
\hline $0.80-0.85$ & 0.0000 & -0.1453 & 0.0063 & -0.1395 & -0.1299 & -0.0131 \\
\hline $0.85-0.90$ & 0.0000 & -0.0884 & 0.0064 & -0.0883 & -0.0880 & -0.0061 \\
\hline $0.90-0.95$ & 0.0000 & -0.0417 & 0.0036 & -0.0417 & -0.0417 & -0.0012 \\
\hline $0.95-0.99$ & 0.0000 & -0.0144 & 0.0000 & -0.0144 & -0.0144 & 0.0000 \\
\hline
\end{tabular}

(d) Minimum Deviation

Table 2. Deviations of the estimation methods 


\section{Conclusions}

The analysis of the experimental data, assuming that a deviation of about 0.01 is acceptable, shows the following results:

1. B\&j method present a very good overall performance and can be used when FR>0.90 but probably this method requires the highest computational effort.

2. M1 method always underestimates the fill rate and it can be used when $F R>0.95$.

3. M2 method always overestimates the fill rate and it can also be used when FR>0.95.

4. M3 method behaves like M1 method but improves its performance so that it can be used when $F R>0.90$.

5. M4 method underestimates but also overestimates the fill rate, but it can also be used when $F R>0.90$.

6. M5 method underestimates and overestimates the fill rate, but its accuracy is the best so that it can be used even when $F R>0.65$.

Therefore, M5 method outperforms the other alternative methods because of its low average deviation, low standard deviation and ease of calculation. In fact this research shows that fill rate can be estimated with high accuracy using a simple method instead of applying the complex calculations needed for the exact method.

\section{Acknowledgement}

This work was supported by the European Regional Development Fund and Spanish Government (MINECO/FEDER, UE) under the project with reference DPI2015-64133-R.

\section{References}

Bijvank, M., Huh, W.T., Janakiraman, G., \& Kang, W. (2014). Robustness of Order-Up-to Policies in LostSales Inventory Systems. Operations Research, 62(5), 1040-1047. https://doi.org/10.1287/opre.2014.1298

Bijvank, M., \& Johansen, S.G. (2012). Periodic review lost-sales inventory models with compound Poisson demand and constant lead times of any length. European Journal of Operational Research, 220(1), 106-114. Elsevier B.V. https://doi.org/10.1016/j.ejor.2012.01.041 
Cardós, M., \& Babiloni, E. (2011). Exact and approximate calculation of the cycle service level in periodic review inventory policies. International Journal of Production Economics, 131(1), 63-68. https://apps.webofknowledge.com/full record.do? product $=$ UA\&search mode $=$ GeneralSearch\&qid $=2 \& S I D=Z 2 C$ mNYYt2gEIRupR3Sg\&page $=1 \&$ doc $=8$ (Accessed: May 2016). https://doi.org/10.1016/j.ijpe.2010.05.012

Cardós, M., Miralles, C., \& Ros, L. (2005). An exact calculation of the cycle service level in a generalized periodic review system. Journal of the Operational Research Society, 57(10), 1252-1255. https://apps.webofknowledge.com/full_record.do? product $=$ UA\&search_mode $=$ GeneralSearch\&qid $=2 \& S I D=Z 2 C m N Y Y t 2 g E I R u p R 3 S g \& p a g e=2 \& d o c=19$

(Accessed: May 2016). https://doi.org/10.1057/palgrave.jors.2602121

Guijarro, E., Cardós, M., \& Babiloni, E. (2012). On the exact calculation of the fill rate in a periodic review inventory policy under discrete demand patterns. European Journal of Operational Research, 218(2), 442-447. http://www.sciencedirect.com/science/article/pii/S0377221711010344 (Accessed: March 2016). https://doi.org/10.1016/j.ejor.2011.11.025

Zipkin, P. (2008a). Old and New Methods for Lost-Sales Inventory Systems. Operations Research, 56(5), 1256-1263. https://doi.org/10.1287/opre.1070.0471

Zipkin, P. (2008b). On the structure of lost-sales inventory models. Operations Research, 56(4), 937-944. https://doi.org/10.1287/opre.1070.0482

\section{Journal of Industrial Engineering and Management, 2016 (www.jiem.org)}

Article's contents are provided on an Attribution-Non Commercial 3.0 Creative commons license. Readers are allowed to copy, distribute and communicate article's contents, provided the author's and Journal of Industrial Engineering and Management's names are included. It must not be used for commercial purposes. To see the complete license contents, please visit http://creativecommons.org/licenses/by-nc/3.0/. 